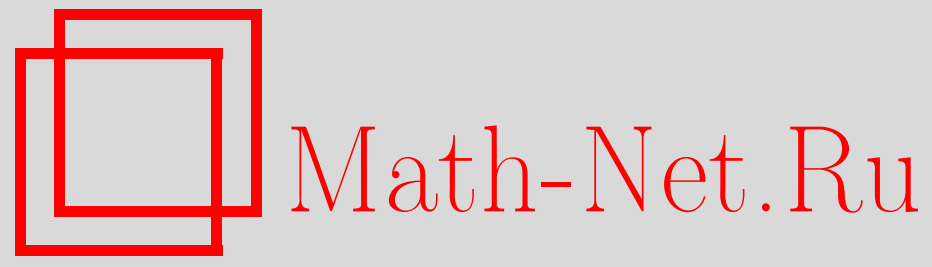

А. В. Лобода, Локальное описание однородных вещественных гиперповерхностей двумерного комплексного пространства в терминах их нормальных уравнений, Функи. анализ и его прил., 2000, том 34, выпуск 2, 33-42

DOI: https://doi.org/10.4213/faa293

Использование Общероссийского математического портала MathNet.Ru подразумевает, что вы прочитали и согласны с пользовательским соглашением

http://www . mathnet.ru/rus/agreement

Параметры загрузки:

IP : 35.173 .219 .149

26 апреля 2023 г., 14:04:24

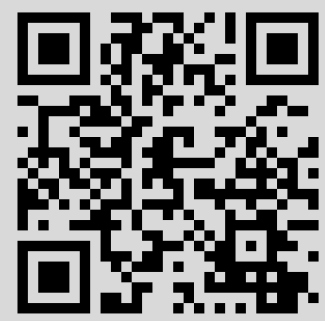


Функциональньй анализ и его приложения

2000, т. 34, вып. 2, с. 33-42

УДК 517.55

\section{Локальное описание однородных вещественных гиперповерхностей двумерного комплексного пространства в терминах их нормальных уравнений}

(C) 2000. А. В. ЛоБОдА

В 1932 г. Э. Картаном [1] были описаны все вещественные гиперповерхности двумерных комплексных пространств, на которых транзитивно действуют группы Ли голоморфных преобразований. В более поздних работах ряда авторов (см., например, [2-4]) также рассматривался с разных точек зрения вопрос об однородных вещественных гиперповерхностях многомерных комплексных пространств. Часть этих работ посвящена двумерному случаю, в то время как построение классификации, аналогичной той, которая приведена в [1], в более высоких размерностях остается пока (насколько известно автору) не решенной в полном объеме задачей.

В работах $[5,6]$ указанное свойство однородности рассматривается при помощи локальных нормальных форм. Данная статья является по сути иллюстрацией эффективности такого подхода. Предлагая в двумерном случае еще одну локальную классификацию однородных поверхностей, мы имеем в виду возможность реализации аналогичных идей в более высоких размерностях.

Заметим, что подобный подход оказывается достаточно эффективным при описании аффинно-однородных поверхностей трехмерного вещественного пространства (см. [7]). В трехмерном комплексном пространстве автором также получены некоторые утверждения об однородности с использованием нормальных форм [8].

Поясним идею такого использования, реализованную ниже. Мы обсуждаем лишь поверхности, голоморфно неэквивалентные сфере, заданные нормальными уравнениями Мозера [9].

Из всех коэффициентов такого уравнения в $§ 2$ выделяется так называемый опорный набор. Его значениями определяются все остальные коэффициенты нормального уравнения однородной несферической поверхности. Затем (§3) этот набор сужается за счет выделения из него существенных коэффициентов, отвечающих за однородность поверхности. Наконец (§4), вычисляя значения коэффициентов опорного набора для известных однородных поверхностей, мы показываем невозможность дальнейшего его сужения.

При построении опорного набора в нашем случае используется ряд утверждений из статьи [6]. В $\S 1$ мы приводим и уточняем некоторые формулы из этой работы.

\footnotetext{
*Работа частично поддержана РФФИ (грант 96-01-01002).
} 
Автор выражает признательность Австралийскому национальному университету (Канберра) за предоставленные возможности для работы над статьей и благодарит А. В. Исаева и В. В. Ежова за плодотворные обсуждения.

\section{$\S 1$. Однородные поверхности и голоморфные векторные поля на них}

Пусть неомбилическая вещественная гиперповерхность $M \in \mathbb{C}^{2}$ задана вблизи начала координат специальным нормальным уравнением Мозера (см. $[9$, p. 247])

$$
\begin{aligned}
v=|z|^{2}+N(z, \bar{z}, u) & =|z|^{2}+\sum_{k+l \geqslant 6, \min (k, l) \geqslant 2} h_{k l}(u) z^{k} \bar{z}^{l} \\
& =|z|^{2}+\sum_{k+l \geqslant 6, \min (k, l) \geqslant 2, m \geqslant 0} N_{k l m} z^{k} \bar{z}^{l} u^{m},
\end{aligned}
$$

в котором $N_{420}=1, N_{430}=0, \operatorname{Re} N_{421}=0$.

В силу однородности гиперповерхности $M$ можно считать, что на ней (вблизи начала координат) транзитивно действует локальная группа Ли локальных голоморфных преобразований. Этой группе соответствует алгебра голоморфных векторных полей

$$
Z=f(z, w) \frac{\partial}{\partial z}+g(z, w) \frac{\partial}{\partial w}
$$

касательных к $M$.

Запишем определяющую функцию поверхности $M$ в виде

$$
\Phi(z, \bar{z}, u, v)=N(z, \bar{z}, u)+|z|^{2}-v .
$$

Тогда условие касания поверхности $M$ полем $Z$ имеет вид

$$
2 \operatorname{Re}\left\{\left.Z(\Phi)\right|_{M}\right\} \equiv 0 .
$$

Левая часть этого тождества представляет собой сумму

$$
\left.\operatorname{Re}(i g+2 f \bar{z})\right|_{v=z \bar{z}+N(z, \bar{z}, u)}+\left.\operatorname{Re}\left(2 f N_{z}+g N_{u}\right)\right|_{v=z \bar{z}+N(z, \bar{z}, u)} .
$$

Ее первое слагаемое изучено в серии статей [9-12], так что большая часть вычислений, необходимых здесь, проделана в этих работах.

Везде ниже мы будем учитывать только выражения, имеющие суммарную степень по $z, \bar{z}$, не превышающую 8 . Следовательно, вместо второго слагаемого в (1.3) можно ограничиться рассмотрением более простого оператора

$$
\left.\operatorname{Re}\left(2 f N_{z}+g N_{u}\right)\right|_{v=z \bar{z}} .
$$

Далее мы будем обсуждать отдельные $(s, \bar{t}, m)$ - и $(s, \bar{t})$-компоненты тождества (1.2), имея в виду полное

$$
H(z, \bar{z}, u)=\sum H_{s \bar{t}, m} z^{s} \bar{z}^{t} u^{m}
$$

и неполное

$$
H(z, \bar{z}, u)=\sum H_{s \bar{t}}(u) z^{s} \bar{z}^{t}
$$

разложения аналитической функции $H=H(z, \bar{z}, u)$ - левой части формулы (1.2). 
Изучение компонент функции $H$, входящих в $\Re$-пространство Мозера (см. [9]), позволяет получить [6, лемма 9] следующее

ПрЕДЛОЖЕНИЕ 1. Если поле $Z=f(z, w) \partial / \partial z+g(z, w) \partial / \partial w$ удовлетворяет условию (1.2), то

$$
\begin{aligned}
& f(z, u)=f_{0}(u)+f_{1}(u) z+2 i \overline{f_{0}^{\prime}(u)} z^{2}+\sum_{k=4}^{\infty}\left(-2 \overline{f_{0}} h_{k 2}(u)\right) z^{k}, \\
& g(z, u)=g_{0}(u)+2 i \overline{f_{0}(u)} z,
\end{aligned}
$$

где набор функиий $f_{0}(u), f_{1}(u), g_{0}(u)$ является решением системь дифференииальньх уравнений

$$
\left\{\begin{array}{l}
f^{\prime \prime}(u)=2 \overline{h_{42}(u) f_{0}(u)} \\
f_{1}^{\prime \prime}(u)=12 \operatorname{Re}\left(f_{0}(u) h_{43}(u)\right), \\
g_{0}^{\prime \prime \prime}(u)=24 \operatorname{Re}\left(f_{0}(u) h_{43}(u)\right)
\end{array}\right.
$$

с начальньцми данньли

$$
\begin{array}{ll}
f_{0}(0)=p \in \mathbb{C}, & f_{0}^{\prime}(0)=a \in \mathbb{C}, \\
f_{1}(0)=\lambda \in \mathbb{C}, & f_{1}^{\prime}(0)=r \in \mathbb{R}, \\
g_{0}(0)=q \in \mathbb{R}, & g_{0}^{\prime}(0)=2 \operatorname{Re}(\lambda), \quad g_{0}^{\prime \prime}(0)=2 r .
\end{array}
$$

Нам понадобятся ниже компоненты типов $(4, \overline{2})$ и $(4, \overline{3})$ тождества $(1.2)$. Отметим, что в работе [6] эти компоненты выписаны с некоторыми неточностями. В исправленной форме $(4, \overline{2})$ - и $(4, \overline{3})$-равенства имеют вид

$$
\begin{aligned}
& \left(10 f_{0}(u) h_{52}(u)+6 \overline{f_{0}(u)} h_{43}(u)\right)+\left(8 f_{1}+4 \overline{f_{1}}\right) h_{42}(u) \\
& +2\left(g_{0}(u) h_{42}^{\prime}-g_{0}^{\prime}(u) h_{42}(u)\right)=0, \\
& 3\left(g_{0}(u) h_{43}^{\prime}(u)-g_{0}^{\prime}(u) h_{43}(u)\right)+\left(2 i f_{0}^{\prime}(u) h_{42}(u)-7 i f_{0}(u) h_{42}^{\prime}(u)\right) \\
& \quad+\left(15 f_{0}(u) h_{53}(u)+12 \overline{f_{0}(u)} h_{44}(u)\right)+\left(12 f_{1}(u)+9 \overline{f_{1}(u)}\right) h_{43}(u)=0 .
\end{aligned}
$$

После подстановки $u=0$ в две последние формулы они существенно упростятся за счет условий

$$
h_{42}(0)=N_{420}=1, \quad h_{43}(0)=N_{430}=0
$$

и примут вид

$(4, \overline{2}, 0)$ :

$$
(3 \lambda+\bar{\lambda})+5 N_{520} p+N_{421} q=0,
$$

И

$$
(4, \overline{3}, 0): \quad 3 q N_{431}+2 i a+\left(15 N_{530}-7 i N_{421}\right) p+12 N_{440} \bar{p}=0
$$

соответственно.

Дифференцируя, далее, $(4, \overline{2})$-соотношение по $u$ при $u=0$, получаем $(4, \overline{2}, 1)$ следствие тождества (1.2) в виде

$$
8 r+10 N_{520} a+10 N_{521} p+6 N_{431} \bar{p}+(8 \lambda+4 \bar{\lambda}) N_{421}+2 N_{422} q=0 .
$$




\section{$\S 2$. Опорный набор коэффициентов для однородной поверхности}

В формулы (1.6)-(1.8) входят параметры $\lambda, a, r, p, q$ и набор

$$
S=\left\{N_{520}, N_{421}, N_{530}, N_{440}, N_{431}, N_{521}, N_{422}\right\}
$$

коэффициентов специального нормального уравнения (1.1) произвольной неомбилической поверхности. Из рассмотрения этих формул легко получается

ПреДЛОЖЕНИЕ 2. Параметры $\lambda, a, r$ выражаются через $p, q$ и коэффициенты из набора $S$.

Набор (2.1) мы будем называть опорньлм для однородной поверхности $M$, имея в виду следующее утверждение.

Теорема 1. Все коэффициенты $N_{k l m}$ нормального уравнения (1.1) однородной неомбилической поверхности $M$ однозначно определяются значениями коэффициентов, входящих в $S$.

Для доказательства рассмотрим сначала $(4, \overline{2})$-компоненту тождества $(1.2)$, т. е. (1.4).

Дифференцируя это тождество по $u$ при $u=0$, мы хотим в первую очередь выразить через набор $S$ все коэффициенты $N_{42(k+1)}, N_{52 k}, N_{43 k}$ при $k \geqslant 2$.

Для этого заметим, что в силу предложений 1 и 2 через $p, q$ и набор $S$ выражаются следующие величины:

$$
\begin{gathered}
f_{0}(0), f_{0}^{\prime}(0), f_{0}^{\prime \prime}(0), f_{0}^{\prime \prime \prime}(0), f_{0}^{(4)}(0), \\
f_{1}(0), f_{1}^{\prime}(0), f_{1}^{\prime \prime}(0), f_{1}^{\prime \prime \prime}(0), \\
g_{0}(0), g_{0}^{\prime}(0), g_{0}^{\prime \prime}(0), g_{0}^{\prime \prime \prime}(0), g_{0}^{(4)}(0) .
\end{gathered}
$$

Тогда вторая производная тождества (1.4) имеет вид

$$
10 f_{0}(u) h_{52}^{\prime \prime}(u)+6 \overline{f_{0}(u)} h_{43}^{\prime \prime}(u)+2 g_{0}(u) h_{42}^{\prime \prime \prime}(u)=D ;
$$

здесь и далее через $D$ мы обозначаем любое выражение, которое при $u=0$ линейно зависит от $p, q$ и полиномиально от $S$. Для всех таких полиномов будем в дальнейшем использовать единое сокращенное обозначение $R(p, q, S)$, а для полиномов, зависящих только от $S$, - обозначение $I(S)$.

В частности, отметим, что все производные из набора (2.1) имеют вид $R(p, q, S)$.

С учетом этих договоренностей последнее уравнение можно переписать (при $u=0)$ в виде

$$
20 p N_{522}+12 \bar{p} N_{432}+12 q N_{423}=R(p, q, S),
$$

или

$$
p\left(20 N_{522}+I(S)\right)+\bar{p}\left(12 N_{432}+I(S)\right)+q\left(12 N_{423}+I(S)\right)=0 .
$$

Так как параметры $p, q$ для однородной поверхности должны быть свободны, из уравнения (2.3) вытекает равенство нулю всех трех коэффициентов при $p, \bar{p}$ и $q$.

Следовательно, $N_{522}, N_{432}$ и $N_{423}$ выражаются через набор $S$. С учетом этого набор производных $(2.2)$, имеющих вид $R(p, q, S)$, можно расширить за счет $f_{0}^{(5)}, f_{1}^{(4)}, g_{0}^{(5)}$.

Продолжая дифференцировать тождество (1.4), получаем индукцией по порядку дифференцирования два утверждения на каждом шаге: 
1) $N_{52 k}, N_{43 k}, N_{42(k+1)}$ выражаются через $S$,

2) $f_{0}^{(k+3)}, f_{1}^{(k+2)}, g_{0}^{(k+3)}$ имеют вид $R(p, q, S)$.

В силу этого коэффициенты $h_{42}(u), h_{52}(u), h_{43}(u)$ специального нормального уравнения однородной поверхности выражаются через опорный набор коэффициентов $S$.

Теперь рассмотрим тождество (1.5), т. е. $(4, \overline{3})$-компоненту тождества (1.2), и запишем его в виде

$$
15 f_{0} h_{53}+12 \overline{f_{0}} h_{44}=\phi\left(h_{42}, h_{43}, f_{0}, f_{1}, g_{0}\right) .
$$

Вследствие предыдущих рассуждений все производные правой части здесь имеют тип $D$. После дифференцирования этого уравнения получим

$$
\left(15 f_{0} h_{53}^{\prime}+12 \overline{f_{0}} h_{44}^{\prime}\right)+\left(15 f_{0}^{\prime} h_{53}+12 \overline{f_{0}^{\prime}} h_{44}\right)=D .
$$

При $u=0$ получаем тогда с учетом предложения 1

$$
p\left(15 N_{531}+I(S)\right)+\bar{p}\left(12 N_{441}+I(S)\right)+q I(S)=0 .
$$

Отсюда, в частности, следует, что $N_{531}$ и $N_{441}$ выражаются через опорный набор $S$. Аналогично последующие дифференцирования тождества (1.5) дадут выражения через $S$ для всех коэффициентов $N_{53 k}, N_{44 k}$ при $k>1$.

Точно так же рассматривается $(5, \overline{2})$-компонента тождества $(1.2)$. В результате такого рассмотрения через $S$ выражается функциональный коэффициент $h_{62}(u)$ из нормального уравнения. Таким образом, все коэффициенты $h_{k l}(u)$, для которых суммарная степень $k+l$ не превосходит 8 , выражаются через $S$.

Далее по индукции можно доказать аналогичное утверждение для всех остальных $h_{k l}(u)$ произвольной суммарной степени $k+l=m>8$.

Эта ссылка на индукцию завершает доказательство теоремы 1.

\section{$\S 3$. Сужение опорного набора}

ТЕорема 2. Имеют место следующие соотношения между коэффициентами специального нормального уравнения

$$
v=|z|^{2}+\sum_{k, l, m} N_{k l m} z^{k} \bar{z}^{l} u^{m}
$$

однородной вещественно-аналитической гиперповерхности в $\mathbb{C}^{2}$ :

$$
\begin{aligned}
& 5 N_{521}-3 \bar{N}_{431}=\frac{15}{2} N_{421} N_{520}-\frac{5}{2} i N_{520}\left(15 N_{530}-7 i N_{421}\right)-30 i N_{440} \bar{N}_{520}, \\
& 24\left(N_{530}+5 \bar{N}_{530}\right)=64 i N_{421}-5\left|N_{520}\right|^{2} \\
& 2 N_{521}+6 \bar{N}_{431}=3 N_{421} N_{520} \\
& 15 N_{531}-7 i N_{422}=\frac{135}{8} N_{520} N_{431}+72 i N_{440}^{2} \\
& \quad-\frac{i}{2}\left(15 N_{530}-5 i N_{421}\right)\left(15 N_{530}-7 i N_{421}\right), \\
& 12 N_{441}+4 i=12 N_{421} N_{440}+\frac{75}{8} N_{431} \bar{N}_{520}+180 N_{440} \operatorname{Im}\left(N_{530}\right), \\
& 8 N_{540}-i N_{431}=6 N_{520} N_{440}-\frac{5}{4} i N_{421} \bar{N}_{520}+\frac{5}{2} N_{530} \bar{N}_{520}, \\
& 2 N_{531}-i N_{422}=2 N_{530} N_{421}+\frac{3}{2} N_{520} N_{431}-i N_{421}^{2} \\
& 5 N_{540}-i N_{431}= \frac{15}{4} N_{520} N_{440}, \\
& N_{441}=0 .
\end{aligned}
$$


Для доказательства этой теоремы рассмотрим однородную неомбилическую поверхность $M$, тождество (1.2) для нее и совокупность $(k, \bar{l}, m)$-следствий из этого тождества.

В силу обсуждений предыдущего параграфа всякое $(k, \bar{l}, m)$-соотношение, кроме самых младших, $(4, \overline{2}, 0),(4, \overline{3}, 0)$ и $(4, \overline{2}, 1)$, принимает вид

$$
p A_{k l m}+\bar{p} B_{k l m}+q C_{k l m}=0 .
$$

При этом вследствие трехмерности рассматриваемой алгебры все коэффициенты $A_{k l m}, B_{k l m}, C_{k l m}$ в этих уравнениях должны равняться нулю.

Напомним, что в $\S 1$ мы уже использовали уравнения (3.11). Для получения формул (3.2)-(3.10) требуется более аккуратное вычисление некоторых из коэффициентов $A_{k l m}, B_{k l m}, C_{k l m}$.

Например, из комплекснозначного $(4, \overline{2}, 1)$-соотношения мы выразили вещественный параметр $r$. Отделяя в $(4, \overline{2}, 1)$-компоненте мнимую часть, мы должны приравнять ее нулю. Получающееся при этом равенство имеет вид (3.11), а коэффициент $A_{421}$ равен в точности левой части уравнения (3.2).

Уравнения $B_{520}=0$ и $C_{520}=0$ превращаются после вычислений в $(3.3)$ и (3.4), а $A_{431}=0$ и $B_{431}=0-$ в $(3.5)$ и (3.6). Уравнения (3.7), (3.8) получаются из равенств $B_{530}=0, C_{530}=0$, а $(3.9),(3.10)-$ из $A_{440}=0, B_{440}=0$ соответственно.

Теперь мы изучим систему (3.2)-(3.10).

Прежде всего, напомним, что коэффициент $N_{421}$ является чисто мнимой величиной в силу определения специальной нормальной формы. Тогда из уравнения (3.3) следует, что $N_{530} \in \mathbb{R}$.

Вводя для упрощения записи новые переменные

$$
\xi=N_{520}, \quad \eta=9 N_{440}, \quad \omega=\operatorname{Im} N_{421},
$$

выразим через них остальные коэффициенты $N_{k l m}$, входящие в исследуемую систему.

Так, например,

$$
N_{530}=-\frac{4}{9} \omega-\frac{5}{144}|\xi|^{2}, \quad 15 N_{530}-7 i N_{421}=\frac{1}{3} \omega-\frac{25}{48}|\xi|^{2},
$$

а уравнения (3.2) и (3.4) примут вид

$$
5 N_{521}-3 \bar{N}_{431}=\frac{20}{3} i \omega \xi+\frac{125}{96} i|\xi|^{2} \xi-\frac{10}{3} i \eta \bar{\xi}, \quad 2 N_{521}+6 \bar{N}_{431}=3 i \omega \xi .
$$

Из этих уравнений находятся $N_{521}$ и $N_{431}$, после чего из формулы (3.5) и (3.8) выражаются через $\xi, \eta, \omega$ коэффициенты $N_{531}$ и $N_{422}$.

При этом формула для $N_{431}$, вытекающая из (3.2) и $(3.4)$, имеет вид

$$
N_{431}=-\frac{5}{108} i \bar{\xi} \omega+\frac{125}{72 \cdot 24} i|\xi|^{2} \bar{\xi}-\frac{5}{27} i \eta \xi .
$$

В свою очередь, уравнения (3.7), (3.9) обсуждаемой системы можно разрешить относительно $N_{540}$ и $N_{431}$, так что

$$
N_{431}=-\frac{25}{108} i \bar{\xi} \omega+\frac{125}{36 \cdot 24} i|\xi|^{2} \bar{\xi} .
$$

Сравнивая выражения (3.12) и (3.13), приходим к уравнению

$$
64 \omega \bar{\xi}-25|\xi|^{2} \bar{\xi}-64 \xi \eta=0 .
$$


Подставляя формулу (3.13) и уравнение (3.10) нашей системы в (3.6), получаем с учетом вещественности коэффициента $N_{530}$

$$
4 i=\frac{12}{9} i \omega \eta+\frac{75}{8} i \bar{\xi}^{2}\left(-\frac{25}{108} \omega+\frac{125}{36 \cdot 24}|\xi|^{2}\right) .
$$

Таким образом, мы сузили опорный набор $S$ до тройки $(\xi, \eta, \omega)$, удовлетворяющей соотношениям (3.14) и (3.15). Эти уравнения являются основой для построения окончательной классификации однородных гиперповерхностей в $\mathbb{C}^{2}$ в терминах коэффициентов их нормальных уравнений.

Для формулировки результата заменим уравнение (3.14) на равносильное, умножая его на $\xi$, и введем еще одно обозначение, полагая $\mu=\frac{25}{24} \xi^{2}$.

Система (3.14), (3.15) примет после этого вид

$$
\left\{\begin{array}{l}
8 \omega|\mu|-3|\mu|^{2}-8 \mu \eta=0 \\
16 \omega \eta-25 \omega \bar{\mu}+15 \bar{\mu}|\mu|=48
\end{array}\right.
$$

Несложно доказать следующее

ПРеДЛОЖЕнИЕ 3. Совокупность решений системь (3.16) имеет вид

$$
\mu=0, \quad \omega \eta=3,
$$

$$
\begin{array}{lll}
16 \omega^{2}-31 \omega \mu+15 \mu^{2}=48, & \eta=\omega-\frac{3}{8} \mu, & \mu \in \mathbb{R}, \mu>0, \\
16 \omega^{2}+31 \omega \mu+15 \mu^{2}=-48, & \eta=-\omega-\frac{3}{8} \mu, & \mu \in \mathbb{R}, \mu<0 .
\end{array}
$$

ЗАмечАниЕ. Геометрически множество всех решений системы (3.16) представляет собой пересечение двух поверхностей в пространстве трех вещественных переменных $\omega, \mu, \eta$. Первая из них есть объединение двух полуплоскостей

$$
\eta=\omega-\frac{3}{8} \mu \quad(\mu>0), \quad \eta=-\omega-\frac{3}{8} \mu \quad(\mu<0)
$$

и разделяющей их плоскости $\mu=0$. Вторая поверхность представляет собой верхнюю $(\mu>0)$ половину однополостного гиперболоида

$$
16 \omega \eta-25 \omega \mu+15 \mu^{2}=48
$$

с присоединенными к ней снизу двумя половинами полостей другого гиперболоида

$$
16 \omega \eta-25 \omega \mu-15 \mu^{2}=48 .
$$

\section{§4. Примеры однородных поверхностей и завершение классификации}

Теорема 3. Каждой точке объединения кривых (3.17)-(3.19) соответствует некоторая однородная гиперповерхность в $\mathbb{C}^{2}$.

ДоКАЗАТЕЛЬСтво. В первую очередь мы сошлемся на результаты работ $[5,13]$. Голоморфный инвариант $\varkappa_{1}$ трубчатой однородной гиперповерхности из [5] пропорционален введенному здесь параметру $\mu$. Каждому положительному значению этого параметра соответствуют ровно две голоморфно различные однородные трубки. Одна из них имеет основанием плоскую степенную кривую $u=x^{s}(-1<s<0)$, а вторая - либо степенную кривую $u=x^{t}$ $(1 / 2<t<1)$, либо логарифмическую спираль $r=e^{a \varphi}$ с некоторым $a>0$, либо кривую $u=x \ln x$, которая является границей между степенными кривыми и спиралями. 
Отрицательным значениям параметра $\mu$ (или $\left.\varkappa_{1}\right)$ соответствуют трубки над степенными кривыми $u=x^{s}(0<s<1 / 2)$.

При рассмотрении случая $\mu=0$ заметим, что равенство $\varkappa_{1}=0$ справедливо для трубок над окружностью $u^{2}+x^{2}=1$ и гиперболой $u^{2}-x^{2}=1$. Здесь мы опишем структуру нормальных уравнений этих поверхностей и нетрубчатых однородных поверхностей [1]

$$
\begin{aligned}
& 1+|z|^{2}+|w|^{2}=a\left|1+z^{2}+w^{2}\right| \quad(a>1), \\
& 1+|z|^{2}-|w|^{2}=a\left|1+z^{2}-w^{2}\right| \quad(a>1), \\
& |z|^{2}+|w|^{2}-1=a\left|z^{2}+w^{2}-1\right| \quad(0<|a|<1) .
\end{aligned}
$$

Заметим, прежде всего, что уравнения

$$
v=\sqrt{1-((z+\bar{z}) / 2)^{2}}, \quad v=\sqrt{1+((z+\bar{z}) / 2)^{2}}
$$

трубок над окружностью и гиперболой являются жесткими (т. е. не зависят от $u=\operatorname{Re} w$ ) и правые части этих уравнений — четные функции (не изменяющиеся при замене $z$ на $-z)$.

ЛЕмма 1. Уравнение любой из поверхностей (4.1)-(4.3) голоморфньлм преобразованием можно привести к жесткому виду $v=F(z, \bar{z})$ с четной функиией $F(z, \bar{z})$.

ДокАЗАтЕЛЬСтво. Легко проверить, что преобразование координат

$$
z^{*}=\frac{w}{\sqrt{1+z^{2}}}, \quad w^{*}=2 \operatorname{arctg} z
$$

превращает уравнение (4.1) в

$$
\operatorname{ch} v=\left(a\left|1+z^{2}\right|-|z|^{2}\right) \quad(a>1),
$$

а уравнение $(4.2)-$ в

$$
\operatorname{ch} v=\left(a\left|1+z^{2}\right|+|z|^{2}\right) \quad(a>1) .
$$

Под действием аналогичного преобразования

$$
z^{*}=\frac{w}{\sqrt{z^{2}-1}}, \quad w^{*}=2 \operatorname{arcth} z
$$

уравнение (4.3) переходит в

$$
\cos v=\left(a\left|1+z^{2}\right|+|z|^{2}\right) \quad(0<|a|<1) .
$$

Так как уравнения (4.5)-(4.7) обладают требуемыми свойствами, лемма 1 доказана.

Предварительные элементарные преобразования (подобные проделанным в [5]) жесткого невырожденного по Леви уравнения $v=F(z, \bar{z})$ приводят его к виду

$$
v=|z|^{2}+\sum_{k, l \geqslant 2} a_{k l} z^{k} \bar{z}^{l} .
$$

При этом в силу четности исходной функции коэффициенты $a_{k l}$ с нечетной суммой $k+l$ равны нулю. Это упрощает процедуру дальнейшей нормализации и, в том числе, приведение к специальной нормальной форме. 
Лемма 2. Пусть вещественная гиперповерхность $M \subset \mathbb{C}^{2}$ задана уравнением (4.8) с четной функцией в правой части и вещественньми коэффициентами $a_{k l}$. Тогда существует мозеровское нормальное уравнение

$$
v=|z|^{2}+\sum h_{k l m} z^{k} \bar{z}^{l} u^{m}
$$

этой поверхности, также обладающее свойством четности. При этом младшие коэффициенты уравнения (4.9) задаются формулами

$$
\begin{gathered}
h_{420}=a_{42}, \quad h_{620}=a_{62}, \quad h_{530}=a_{53}-4 a_{22} a_{42}, \\
h_{440}=a_{44}-4 a_{22} a_{33}+\frac{10}{3} a_{22}^{3}, \quad h_{421}=i a_{22} a_{42} .
\end{gathered}
$$

Доказательство этой леммы является чисто техническим и совпадает в своих первых шагах с аналогичными рассмотрениями из [5]. Остальные детали мы здесь не приводим.

Переход от нормального уравнения (4.8) с ненулевым вещественным коэффициентом $h_{420}$ к специальному нормальному можно осуществить заменой

$$
z \rightarrow\left|h_{420}\right|^{-1 / 4} z, \quad w \rightarrow\left|h_{420}\right|^{-1 / 2} w
$$

при положительном $h_{420}$ или

$$
z \rightarrow(-i)\left|h_{420}\right|^{-1 / 4} z, \quad w \rightarrow\left|h_{420}\right|^{-1 / 2} w,
$$

если $h_{420}$ отрицательно.

При этом коэффициенты $N_{k l m}$ будут задаваться формулами

$$
\begin{gathered}
N_{620}=\frac{a_{62}}{\left|a_{42}\right|^{3 / 2}}, \quad N_{530}=\frac{\left(a_{53}-4 a_{22} a_{42}\right) \operatorname{sgn}\left(a_{42}\right)}{\left|a_{42}\right|^{3 / 2}}, \\
N_{440}=\frac{a_{44}-4 a_{22} a_{33}+\frac{10}{3} a_{22}^{3}}{\left|a_{42}\right|^{3 / 2}}, \quad N_{421}=i \frac{a_{22} a_{42} \operatorname{sgn}\left(a_{42}\right)}{\left|a_{42}\right|^{3 / 2}}=i \frac{a_{22}}{\left|a_{42}\right|^{1 / 2}} .
\end{gathered}
$$

Вернемся теперь к конкретным уравнениям (4.5)-(4.7). Коэффициенты уравнения вида (4.8) для первой из описываемых ими поверхностей равны

$$
\begin{aligned}
a_{22} & =\frac{3}{4} \frac{a}{\left(a^{2}-1\right)}, & a_{42} & =-\frac{3}{16} \frac{a}{\left(a^{2}-1\right)^{2}}, \\
a_{62} & =-\frac{1}{32} \frac{a}{\left(a^{2}-1\right)^{3}}, & a_{33} & =\frac{1}{6} \frac{5 a^{2}+1}{\left(a^{2}-1\right)^{2}}, \\
& =-\frac{1}{2} \frac{a^{2}}{\left(a^{2}-1\right)^{3}}, & a_{44} & =\frac{1}{64} \frac{a\left(70 a^{2}+33\right)}{\left(a^{2}-1\right)^{3}} .
\end{aligned}
$$

Тогда, согласно формулам (4.11),

$$
N_{440}=\frac{1}{3 \sqrt{3} \sqrt{a}}, \quad N_{421}=i \sqrt{3} \sqrt{a} \quad(a>1) .
$$

Ясно, что эта пара коэффициентов удовлетворяет уравнению (3.17). После аналогичных вычислений для поверхностей (4.6) параметр $\omega=\operatorname{Im} N_{421}$ оказывается равным $-\sqrt{3} \sqrt{a}$.

Наконец, для поверхностей (4.7) имеем

$$
\omega=\operatorname{Im} N_{421}=\sqrt{3} \sqrt{|a|} \operatorname{sgn}(a) .
$$

Учитывая пределы изменения параметра $a$ в уравнениях (4.5)-(4.7), видим, что областью значений параметра $\omega=\operatorname{Im} N_{421}$ для этих поверхностей является вся вещественная ось за исключением трех точек: $-\sqrt{3}, 0, \sqrt{3}$. 
Значения $-\sqrt{3}$ и $\sqrt{3}$ параметр $\omega$ принимает на трубчатых поверхностях над окружностью и гиперболой, так как для них вычисления дают

$$
\begin{aligned}
& a_{22}=\frac{3}{4}, \quad a_{42}=\frac{3}{16}, \quad a_{33}=1, \quad a_{62}=-\frac{1}{32}, \quad a_{53}=\frac{1}{2}, \quad a_{44}=\frac{103}{64}, \\
& a_{22}=-\frac{3}{4}, \quad a_{42}=\frac{3}{16}, \quad a_{33}=1, \quad a_{62}=\frac{1}{32}, \quad a_{53}=-\frac{1}{2}, \quad a_{44}=-\frac{103}{64}
\end{aligned}
$$

соответственно. Значение же $\omega=0$ запрещается предложением 3. Теорема 3 доказана.

Эта теорема завершает классификацию однородных неомбилических гиперповерхностей пространства $\mathbb{C}^{2}$ в терминах тройки чисел $\left(N_{520}^{2}, N_{440}, N_{421}\right)$. В то же время любая поверхность с ненулевым $N_{520}$ допускает два различных специальных нормальных уравнения [9]. Поэтому можно говорить о классификации однородных неомбилических поверхностей в терминах именно коэффициентов таких уравнений.

ЗАмечАниЕ. Вся совокупность однородных гиперповерхностей в $\mathbb{C}^{2}$ содержит помимо поверхностей рассмотренного типа лишь сферу и вещественную гиперплоскость.

\section{ЛИТЕРАТУРА}

1. Cartan E. Sur la geometrie pseudoconforme des hypersurfaces de deux variables complexes. Ann. Math. Pura Appl., (4) 11 (1932), 17-90 (Oeuvres II, 2, 1231-1304).

2. Morimoto A., Nagano T. On pseudo-conformal transformations of hypersurfaces. J. Math. Soc. Japan, 15, No. 3, 289-300 (1963).

3. Rossi $H$. Homogeneous strongly pseudoconvex hypersurfaces. Rice Univ. Studies, 59, No. 3, 131-145 (1973).

4. Ehlers F., Neumann W. D., Scherk J. Links of surface singularities and CR space forms. Comment. Math. Helv., 62, 240-264 (1987).

5. Лобода А. В. О некоторых инвариантах трубчатых гиперповерхностей в $\mathbb{C}^{2}$. Матем. заметки, 59, № 2, 211-223 (1996).

6. Белошапка В. К. Однородные гиперповерхности в $\mathbb{C}^{2}$. Матем. заметки, 60 , № 5 , 760-764 (1996).

7. Лобода А. В. О классификации аффинно-однородных поверхностей пространства $\mathbb{R}^{3}$ в терминах их нормальных уравнений. Понтрягинские чтения-IX, Тезисы докладов, Воронеж, 1998, с. 124.

8. Лобода А. В. О транзитивных действиях групп на слабо однородных многообразиях. Понтрягинские чтения-VIII, Тезисы докладов, Воронеж, 1997, с. 92.

9. Chern S. S., Moser J. K. Real hypersurfaces in complex manifolds. Acta Math., 133, No. 3, 219-271 (1974).

10. Белошапка B. K. О размерности групп автоморфизмов аналитической гиперповерхности. Изв. АН СССР, сер. матем., 43, № 2, 243-266 (1979).

11. Лобода А. В. О локальных автоморфизмах вещественно-аналитических гиперповерхностей, Изв. АН СССР, сер. матем., 45, № 3, 620-645 (1981).

12. Лобода $A$. В. Линеаризуемость автоморфизмов несферических поверхностей. Изв. АН СССР, сер. матем., 46, № 4, 864-880 (1982).

13. Гузеев Р. Н., Лобода А. В. О голоморфных инвариантах логарифмических спиралей. Изв. ВУЗов. Математика, № 2, 16-19 (1998). 